\title{
E-BOSS: an Extensive stellar BOw Shock Survey
}

\section{Methods and first catalogue}

\author{
C. S. Peri ${ }^{1,2}$, P. Benaglia ${ }^{1,2}$, D. P. Brookes ${ }^{3}$, I. R. Stevens ${ }^{3}$, and N. L. Isequilla ${ }^{2}$ \\ ${ }^{1}$ Instituto Argentino de Radioastronomía, CCT-La Plata (CONICET), C.C.5, 1894 Villa Elisa, Argentina \\ e-mail: cperi@fcaglp.unlp.edu.ar \\ 2 Facultad de Ciencias Astronómicas y Geofísicas, UNLP, Paseo del Bosque s/n, 1900 La Plata, Argentina \\ 3 School of Physics and Astronomy, University of Birmingham, Edgbaston, Birmingham B15 2TT, UK
}

Received 17 September 2011 / Accepted 25 November 2011

\section{ABSTRACT}

\begin{abstract}
Context. Bow shocks are produced by many astrophysical objects where shock waves are present. Stellar bow shocks, generated by runaway stars, have been previously detected in small numbers and well-studied. Along with progress in model development and improvements in observing instruments, our knowledge of the emission produced by these objects and its origin can now be more clearly understood.

Aims. We produce a stellar bow-shock catalogue by applying uniform search criteria and a systematic search process. This catalogue is a starting point for statistical studies, to help us address fundamental questions such as, for instance, the conditions under wich a stellar bow shock is detectable.

Methods. By using the newest infrared data releases, we carried out a search for bow shocks produced by early-type runaway stars. We first explored whether a set of known IRAS bow shock candidates are visible in the most recently available IR data, which has much higher resolution and sensitivity. We then carried out a selection of runaway stars from the latest, large runaway catalogue available. In this first release, we focused on OB stars and searched for bow-shaped features in the vicinity of these stars.

Results. We provide a bow-shock candidate survey that gathers a total of 28 members, which we call the Extensive stellar BOw Shock Survey (E-BOSS). We derive the main bow-shock parameters, and present some preliminary statistical results on the detected objects. Conclusions. Our analysis of the initial sample and the newly detected objects yields a bow-shock detectability around OB stars of $\sim 10$ per cent. The detections do not seem to depend particularly on either stellar mass, age or position. The extension of the E-BOSS sample, with upcoming IR data, and by considering, for example, other spectral types as well, will allow us to perform a more detailed study of the findings.
\end{abstract}

Key words. infrared: ISM - infrared: stars - stars: early-type - catalogs

\section{Introduction}

Many astrophysical objects perturb the interstellar medium (ISM) and produce different kinds of observable structures. Early-type stars with large peculiar velocities (termed runaway stars) are one example of the perturbing agent. They are responsible for the generation of stellar bow shocks.

Runaway stars have been studied for several decades. There are currently two proposed mechanisms for the origin of their high velocities (Hoogerwerf et al. 2000). One is the binary supernova scenario (BSS, Zwicky 1957; Blauuw 1961) and the other is the dynamical ejection scenario (DES, Poveda et al. 1967; Gies \& Bolton 1986). In the BSS, the runaway star that was originally part of a binary system, acquires a high speed when its companion explodes as a supernova. In the context of the DES, the runaway star achieves its velocity thanks to the dynamical interaction with one or more stars. In some cases, the trajectory of the stars and the original systems can be reconstructed, and the mechanism that has kicked the star can be identified. The gathered evidence has not always enabled strong conclusions to be made (see for instance the dissenting findings by Comerón \& Pasquali 2007; Gvaramadze \& Bomans 2008). Studies such as Moffat et al. (1998) of runaway stars suggest that the BSS is slightly more likely, and that those stars with significant peculiar supersonic motion relative to the ambient ISM, tend to form bow shocks in the direction of the motion.

Abundant studies of individual or smaller groups of runaway stars can be found. However, compilations of large number of high velocity stars are scarce. A good example is the Galactic O-Star Catalog (GOSC, Maíz Apellániz et al. 2004), which contains the physical properties of about 40 of these stars. Tetzlaff et al. (2010) carried out an extensive kinematical and probability study, that allowed us to identify thousands of runaway stars. The authors used the Hipparcos catalogue (Perryman et al. 1997) and built a database of $\sim 2500$ objects.

The effects of runaway stars on the environment and the interaction between those stars and the ISM have been widely documented. A fundamental contribution can be found in Van Buren \& McCray (1988), who presented a list of 15 bow shock-like candidates, found while studying Galactic HII regions detected in IRAS (InfraRed Astronomical Satellite) data. Their study triggered an extended search for stellar bow-shock candidates, which resulted in almost 60 similar sources of which around 20 were actually candidates (Van Buren et al. 1995; NoriegaCrespo et al. 1997: NC97). Some of the candidates were studied by Brown \& Bomans (2005), using the $\mathrm{H} \alpha$ all-sky survey (SHASSA/VTSS). They searched images of 37 objects of the list of Van Buren et al. (1995) and detected 8 bow shocks. They 
also calculated environmental parameters and in all the cases they found consistency with the features of the warm ionized medium.

In the past few years, searches of relatively small sky regions have revealed more stellar bow shocks. Povich et al. (2008) reported the discovery of six stellar bow shocks in the starforming regions M 17 and RCW 49 from Spitzer GLIMPSE (Galactic Legacy Infrared Mid-Plane Survey Extraordinaire) images. By combining 2MASS (Two Micron All-Sky Survey), Spitzer, MSX (Midcourse Space eXperiment), and IRAS data, they obtained the SEDs of the bow shocks and stars associated with them. Other 10 bow-shock candidates were found by Kobulnicky et al. (2010) using mid-IR images from the Spitzer Space Telescope Cygnus X Legacy Survey. Arnal et al. (2011) exposed a study of the surrounding emission related to the star HD 192281, a member of the OB association Cygnus OB8. They analyzed neutral hydrogen, radio continuum, molecular gas (CO) emission and infrared (IR) data, and derived several parameters of the medium. They concluded that HD 192281 seems to generate a stellar bow shock and it is possible that triggered star formation is underway. Bow shocks have been seen around some massive X-ray binaries, such as Vela X-1 (Kaper et al. 1997) and 4U 1907+09 (Gvaramadze et al. 2001a).

Gvaramadze et al. (2011b) carried out a search for OB stars running away from the young stellar cluster NGC 6357. They discovered seven bow shocks, and thoroughly discussed the scenario for these structures to develop.

During the investigation of the outskirts of Cygnus OB2, Comerón \& Pasquali (2007) discovered a bow-shaped structure close to the high mass runaway star $\mathrm{BD}+43^{\circ} 3654$. From spectroscopic data, they re-classified the star and derived its stellar mass, ranking the star as one of the three most massive runaways known in the Milky Way. Stellar proper motions as well as MSX observations confirmed that the bow shock could be produced by the star, in turn probably a former member of the mentioned association. In a subsequent publication, Benaglia et al. (2010) carried out a study of the bow shock produced by $\mathrm{BD}+43^{\circ} 3654$, and evaluated whether the structure could give rise to high energy emission. By means of dedicated VLA observations, they calculated the spectral index in the region of the bow shock and measured values compatible with a non-thermal radiation origin. Assuming that there is a population of relativistic particles, they estimated the SEDs generated by different radiative processes.

In spite of these studies, numerous questions remain to be answered. How common are stellar bow shocks? In which conditions are they produced and detected? Questions such as how the spectral type, velocity, stellar wind, coordinates of the star, and other parameters influence the formation of a bow shock will be more clearly addressed as more examples are analyzed.

In this paper, we introduce the Extensive BOw Shock Survey (E-BOSS) generated by means of on-line available data from different missions. We used the first-release Wide-field Infrared Survey Explorer (WISE) images, as a tool to detect new structures and to contribute to what is known about those already studied.

In the next section, we briefly discuss the theoretical background and analyze the conditions that could increase the probability of detecting a stellar bow shock. Section 3 describes the procedure followed to obtain the sample in which we search for bow-shock candidates. In Sect. 4, we present the bow shocks found and characterize them. Section 5 shows the statistics performed, and Sect. 6 has a corresponding discussion. In the last section, we comment on the immediate prospects.

\section{Theoretical considerations}

Runaway stars move through the ISM with velocities that overcome the field stars velocities and the sound of speed in the ISM. They shock the ISM and produce the so-called bow shocks that sweep up the ISM matter into thin, dense shells (Wilkin 1996). The theoretical study of bow shocks has been developed not only from an analytical point of view but also with numerical simulations.

Wilkin (1996) derived analytical exact solutions for stellar wind bow shocks in the thin-shell limit, stressing the importance of the conserved momentum within the shell. He developed a simple method to reproduce the shape of the shell, mass column, and velocity of the shocked gas throughout the shell. Later, Wilkin (2000) improved the model studying the modifications of the bow shocks in two cases where: (i) a star moves supersonically with respect to an ambient medium with a density gradient perpendicular to the stellar velocity, and (ii) a star with a mis-aligned, axisymmetric wind moves in a uniform medium. He found that the region of the stand-off point (where ambient and wind pressures balance each other) is tilted in both cases. In that way, the star does not lie in the line that divides the bow shock into two halves.

Dgani et al. (1996a,b) peformed a stability analysis of thin isothermal bow shocks. In the first paper, they showed that the bow shocks produced by stars with fast stellar winds are more stable than those generated by slow winds. In the second article, the authors then investigated non-linear instabilities and run numerical simulations to solve the problem. They proposed that the slower the wind, the highest the instability. They also applied the model to the star $\alpha$ Cam and concluded that the clumps observed might be explained by the instabilities.

Comerón \& Kaper (1998) conducted a semi-analytical study of bow shocks produced by OB runaway stars and derived expressions that suggest different results from those obtained with the assumption of instantaneous cooling of the shocked gas (Wilkin 1996). They ran numerical simulations that reveal a wealth of details in the formation, structure, and evolution of the bow shocks. These features strongly depend on the conditions of the medium and star. The bow shocks can either form or not; and if they do form, they can be either stable, unstable, or layered.

To complete the study of bow shocks and improve the models, they need to be observed and analyzed. In principle, owing to the presence of the shocks that the runaway stars produce in the ISM, the dust is heated and in that way re-radiates at infrared wavelengths. As we describe in other sections, bow shocks have been observed at infrared (e.g. van Buren et al. 1995, NC97), optical (e.g. Brown \& Bomans 2005), and - in a few cases - radio wavelengths, and will eventually be detected at high energy waves (Benaglia et al. 2010).

\section{The making of the E-BOSS sample}

We searched for stellar bow shocks in various different databases described later. We account for a number of criteria that help us to identify new stellar bow shocks.

1. We examined the surroundings of early-type runaway stars, as these have high velocities and strong winds that sweep up interstellar matter and also high luminosities that contribute to the dust heating.

2. We selected nearby stars $(d<3 \mathrm{kpc})$, which thus have brighter bow shocks. 
Table 1. Star members of Group 1.

\begin{tabular}{lccccc}
\hline \hline HIP & HD/BD/Other & Spectral type & MSX & WISE & 1997 \\
\hline 1415 & 1337 & O9IIInn+.. & - & - & \\
2599 & $2905 / \kappa$ Cas & B1Iae & $\star$ & $\supset$ & $\supset$ \\
3478 & 4142 & B5V & - & $\star$ & $\bullet$ \\
13296 & 17505 & O6Ve & $\star$ & $\bullet *$ & $\bigcirc ?$ \\
14514 & 19374 & B1.5V & - & $\star$ & $\bullet$ \\
15063 & 19820 & O8.5III & $\star$ & $\star$ & $\supset ?$ \\
17358 & 22928 & B5III & - & $\supset$ & $\supset$ \\
18370 & 24431 & O9IV-V & $\times$ & $\star$ & $\bullet$ \\
22783 & $30614 / \alpha$ Cam & O9.5Iae & - & $\supset$ & $\supset$ \\
24575 & 34078 & O9.5Ve... & - & $\supset$ & $\bullet$ \\
25947 & +391328 & O9III: & $\times$ & $\star$ & $\bullet$ \\
& $36862 / \lambda$ Ori & B0.5V & - & {$[\mathrm{a}]$} & $\supset$ \\
26220 & 37020 & B0.5V & $\odot$ & $\odot$ & $\bullet$ \\
26889 & 37737 & B0II: & $\times$ & $\bullet *$ & $\bigcirc ?$ \\
28881 & 41161 & O8V & - & $\supset$ & $\supset$ \\
29147 & 41997 & O7.5V & - & $\odot$ & $\bullet$ \\
29276 & $42933 / \delta$ Pic & B3III+... & - & $\supset$ & $\supset$ \\
31978 & 47839 & O7Ve & $\star$ & $\odot$ & \\
32067 & 48099 & O6e & $\star$ & $\supset$ & $\supset$ \\
33836 & 52533 & O9V & $\star$ & $\bullet$ & $\bullet$ \\
34536 & 54662 & O7III & $\star$ & $\supset$ & $\supset$ \\
35415 & $57061 / \tau$ CMa & O9Ib & - & $\odot$ & $\supset$ \\
38430 & 64315 & O6e & $\supset$ & $\odot$ & $\bullet$ \\
39429 & 66811 & O4If(n)p & - & - & $\bullet$ \\
50253 & 89137 & O9.5III(n)p & $\times$ & - & $\bullet$ \\
\hline 56726 & 92206 & O6.5V & {$[\mathrm{b}]$} & - & $\bullet$ \\
63117 & 101131 & O6V((f)) & - & $\odot$ & $\bullet$ \\
\hline & 112244 & O9Ibe & - & $\bullet$ & $\bullet$ \\
\hline
\end{tabular}

\begin{tabular}{|c|c|c|c|c|c|}
\hline HIP & HD/BD & Spectral type & MSX & "WISE & 1997 \\
\hline 72510 & 130298 & O5/O6 & $\star$ & J & J \\
\hline 74778 & 135240 & $08.5 \mathrm{~V}$ & - & - & - \\
\hline 77391 & 329905 & $\mathrm{O}+\ldots$ & $x$ & $\supset$ & - \\
\hline 78401 & $143275 / \delta$ Sco & B0.2IVe & - & $\supset$ & $\supset$ \\
\hline 81377 & 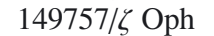 & $\mathrm{O} 9 \mathrm{~V}$ & - & $\supset$ & $\supset$ \\
\hline 84588 & 156212 & $\mathrm{O}+\ldots$ & $\star$ & $\star$ & \\
\hline $85569 \dagger$ & 158186 & $09.5 \mathrm{~V}$ & $\star$ & - & - \\
\hline $88333 \dagger$ & 164492 & O6 & - & $\bullet *$ & - \\
\hline 90320 & 169582 & O5e & $x$ & - & - \\
\hline 91113 & 171491 & B5 & $\star$ & $\odot$ & $\supset$ \\
\hline 92865 & 175514 & O8:Vnn & $\star$ & $\supset$ & $\supset$ \\
\hline 97280 & 186980 & O7.5III... & $x$ & $\phi$ & - \\
\hline 97796 & 188001 & O7.5Ia... & - & כ & $\supset$ \\
\hline 98418 & 227018 & O6.5III & $\odot$ & - & - \\
\hline 98530 & 189957 & B0III & - & - & - \\
\hline 101186 & 195592 & O9.5Ia & $\supset$ & - & $\supset$ \\
\hline & +43 3654/U824 & O4I & $\supset$ & - & $\supset$ \\
\hline 103371 & 199579 & O6V((f)) & $\star$ & - & - \\
\hline 104642 & 202214 & BOII & - & - & - \\
\hline 105186 & 203064 & O8e & $\star$ & - & - \\
\hline 105268 & 203467/6 Cep & B3IVe & - & - & כ \\
\hline 107598 & 207538 & O9V & $\bullet$ & - & $\bullet$ \\
\hline 109556 & 210839/入 Сер & O6If(n)p & $\star$ & - & כ \\
\hline 110609 & 212593 & B9Iab & - & - & $\bullet$ \\
\hline 110817 & 213087 & B0.5Ibe... & - & - & - \\
\hline 111841 & 214680 & O9V & - & - & $\supset ?$ \\
\hline 114990 & +631964 & BOII & $\star$ & $\supset$ & - \\
\hline 117957 & 224151 & B0.5II-III & - & $\star$ & - \\
\hline
\end{tabular}

Notes. Stars of group 1 were taken from Noriega Crespo et al. (1997). All were observed by the IRAS satellite and two by the Spitzer-Glimpse program $(\dagger)$. The first column lists the Hipparcos number of the star; the second one, other identification(s). Column (3) shows the spectral classification, from the GOSC whenever possible or from NC97 otherwise. Columns (4) and (5) give information about MSX and WISE emission on the stellar fields, according to the following symbols. $\star$ : Point source on star, $\supset$ : bow shock candidate, $\supset$ ?: doubtful bow shock candidate, -: stellar field not covered by the survey, $\times$ : no star or emission close-by, $\odot$ : extended source on star, $\bullet *$ : confusion with larger structure, $\phi$ : diffuse emission, $\bullet$ : emission excess, $\bigcirc$ ?: possible bubble. Column (6) lists the qualifiers (same meaning as before) as in the original work by NC97. [a] The star HD 36862, together with HD 36861 (O8III), belong to a rich cluster, are not single, and have a large spatial velocity. More than one feature can produce the surrounding IR emission detected. [b] Cappa et al. (2008) studied the environs of HD 92206, and identified an HII region. We discarded these two cases from our sample.

We generated the initial sample in two different ways. We considered first bow shock candidates that had previously been detected in the IRAS data (NC97). Secondly, we carried out a systematic search around runaway stars that could produce bow shocks, using the catalogue of Tetzlaff et al. (2010). The next section describes the process in more detail. In the rest of the paper, we divide the E-BOSS sample into two groups, groups 1 and 2.

\subsection{Group 1}

For this group, we took the bow shock candidates database of NC97. These authors searched for bow shocks and other features at IR wavelengths from the HiReS-IRAS maps. Table 1 lists the 56 objects that are OB stars from NC97, hereafter called group 1 (the WR stars HD 50896 and HD 192163, from the original list, were excluded).

\subsection{Group 2}

The second group was extracted from the catalogue of Tetzlaff et al. (2010). From a sample of 7663 young stars observed by Hipparcos (Perryman et al. 1997), the authors built a catalogue of 2546 runaway stars candidates. Their study lists stellar names, velocities, spectral types, ages, and masses. From this last list, we found 244 stars of spectral type O to B2. We list this 244 stars in Table 2; they comprise what we call hereafter group 2. A total of 17 stars are common to both groups.

\subsection{Information collation}

We used the NASA/IPAC Infrared Science Archive ${ }^{1}$ to gather relevant infrared and sub-millimeter missions data (IRAS, MSX, WISE, Spitzer, etc.). In practice, data from MSX and WISE ${ }^{2}$ have proven to be the most useful. In particular, WISE has discovered various bow shocks. Although the published WISE data covers half of the sky, in effect, it covers more than two thirds of both group 1 and group 2 samples. When further data becomes available, it will be added to a subsequent release of the E-BOSS sample.

In addition to the infrared data, we searched for bow shock emission at $\mathrm{H} \alpha$, using the Virginia Tech. Spectral Survey (VTSS, Dennison et al. 1997) and the Southern Hemispheric H $\alpha$ Sky Survey Atlas (SHASSA, Gaustad et al. 2001). These surveys cover most of the sky with a spatial resolution of $0.8^{\prime}$ for SHASSA and $1.6^{\prime}$ for VTSS. We did not find any convincing bow shock candidates for either our group 1 or group 2 sample.

\footnotetext{
${ }^{1}$ http://irsa.ipac.caltech.edu/

2 http://wise.ssl.berkeley.edu/
} 

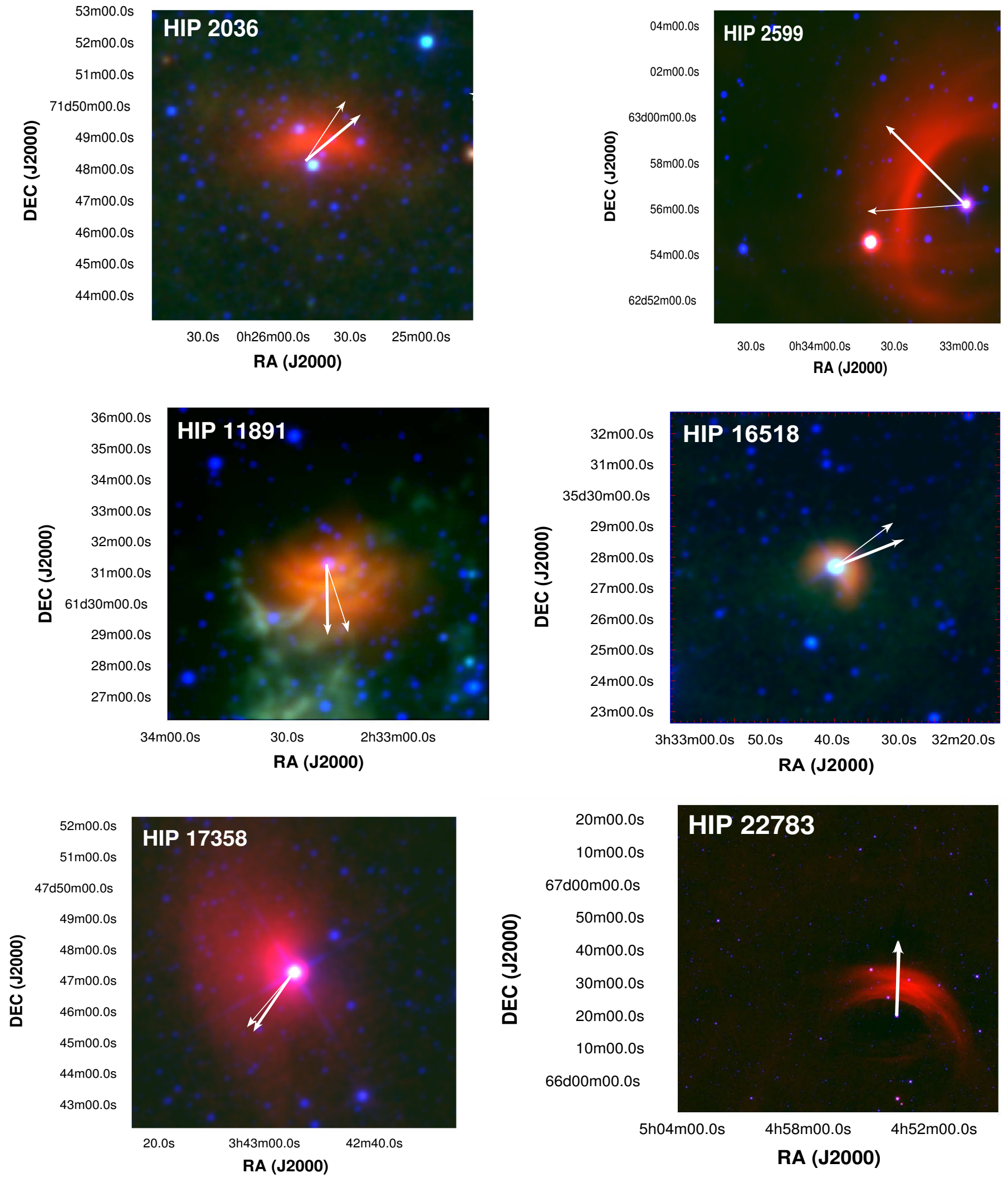

Fig. 1. WISE images of the bow shock candidates around 6 OB stars. Color mapping: blue $=3.4$ microns; green $=12.1$ microns; red $=22.2$ microns . The color scales are in data numbers (DNs); the WISE data is not calibrated in their surface brightness. The vectors indicate the direction of the star proper motion: the thicker one represents the one derived from Hipparcos data by van Leeuwen (2007); the thinner one is the same but corrected for the ISM motion caused by Galactic rotation. The vectors length are not scaled with the original values. 
C. S. Peri et al.: The extensive stellar BOw shock survey
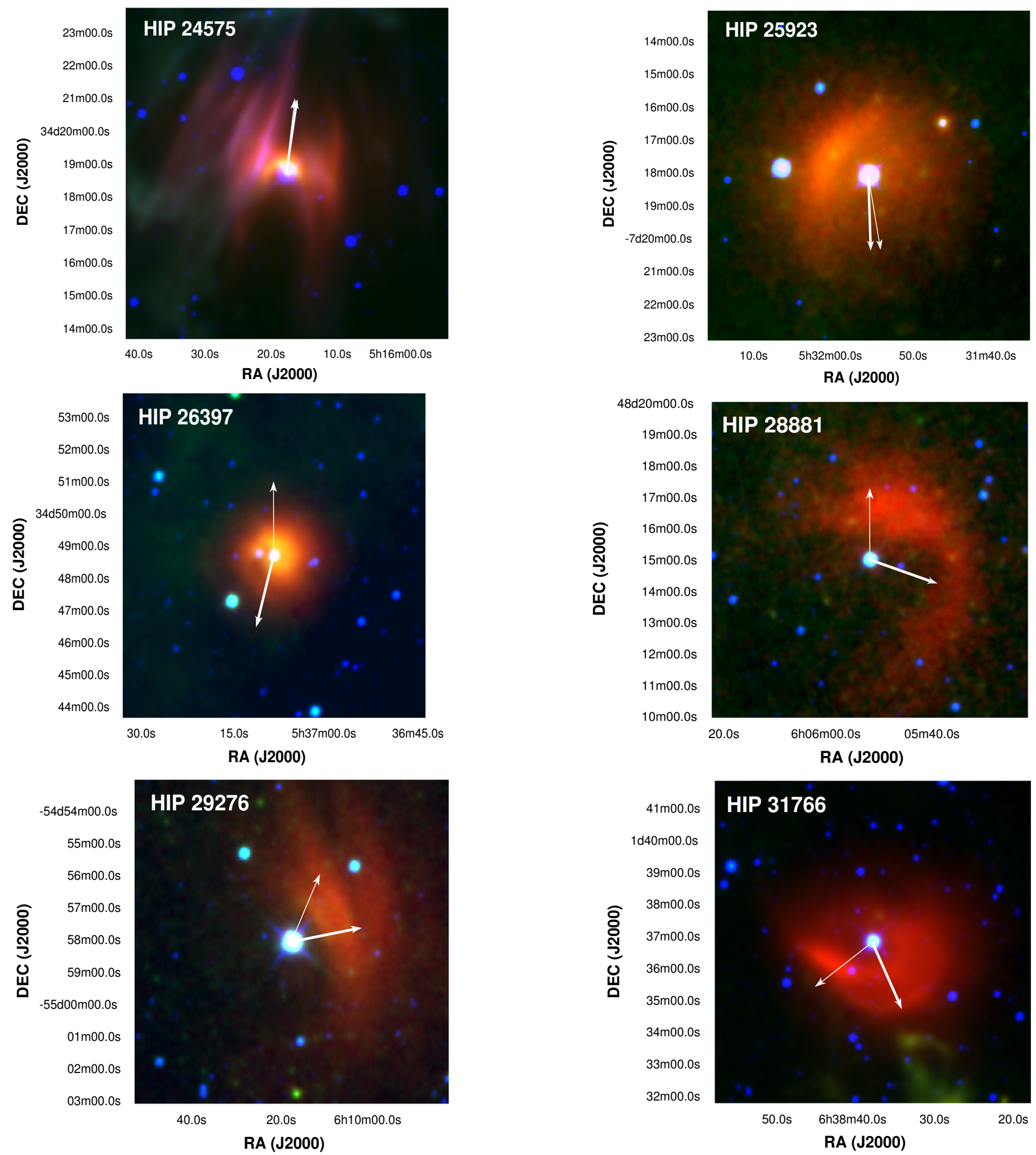

Fig. 2. Same as in Fig. 1, for another six OB stars.

This is in contrast to Brown \& Bomans (2005), who found 8 possible bow shock candidates, starting with a target list of 37 stars from van Buren et al. (1995). We note that the stars are often located in complex $\mathrm{H} \alpha$ emission regions making identification of a bow shock feature difficult. In some cases, we did detect possible $\mathrm{H} \alpha$ emission from a bow shock but which is not coincident with a clear bow shock detected by WISE (for example,
HD 48099 and HD 149757). In these cases, we relied on the infrared detection.

The detection of radio emission and the measurement of a non-thermal radio spectral index coincident with the location of the bow-shock candidate related to the star BD $+43^{\circ} 3654$ encouraged us to check for low frequency radio emission around the E-BOSS members. We used the postage stamp server for 

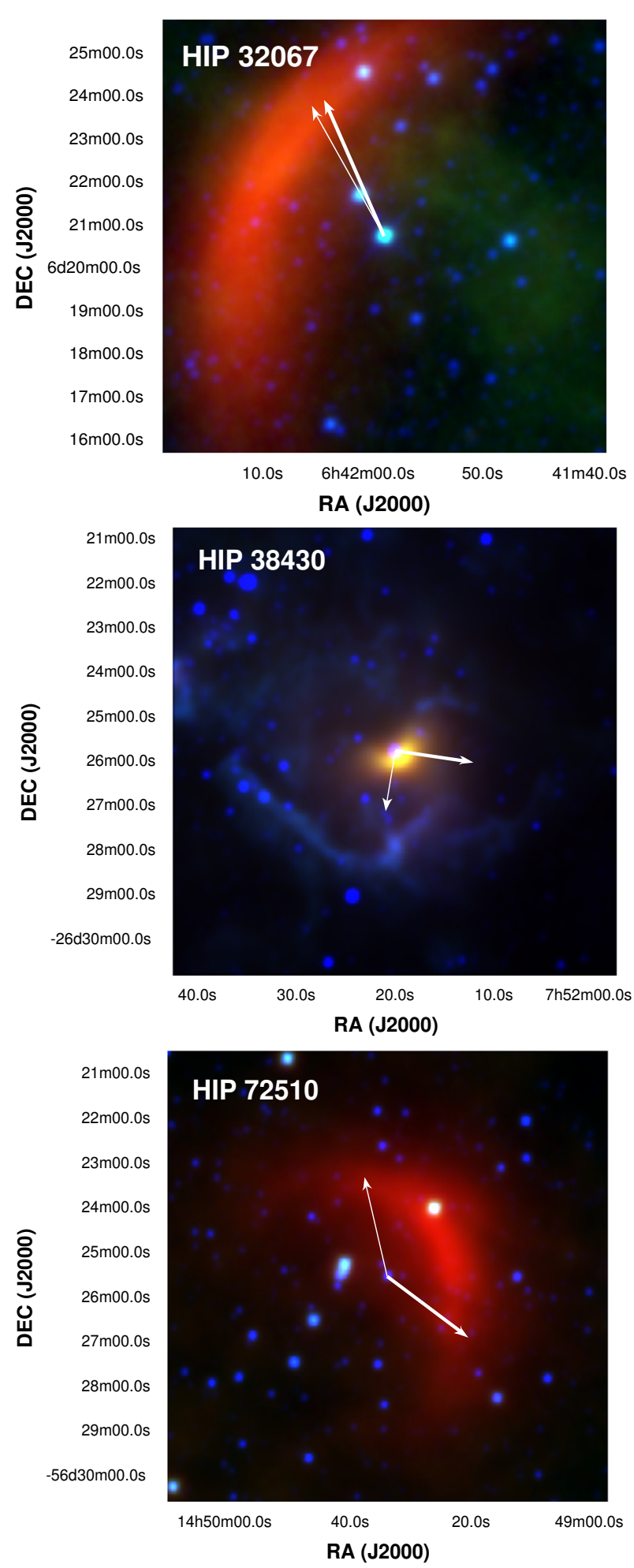

Fig. 3. Same as in Figs. 1 and 2, for another six OB stars.

NRAO/VLA Sky Survey (NVSS, Condon et al. 1998), which returns radio images of the sky in various formats. Most of the radio maps contain only point sources, which are not particularly related to the IR features. However, three E-BOSS objects, apart from $\mathrm{BD}+43^{\circ} 3654$, correspond interesting comma-shaped radio sources at $1.4 \mathrm{GHz}$. A thorough investigation based on dedicated radio observations towards these candidates, HIP 88652 , HIP 38430, and HIP 11891 is under way (Peri et al., in prep.).
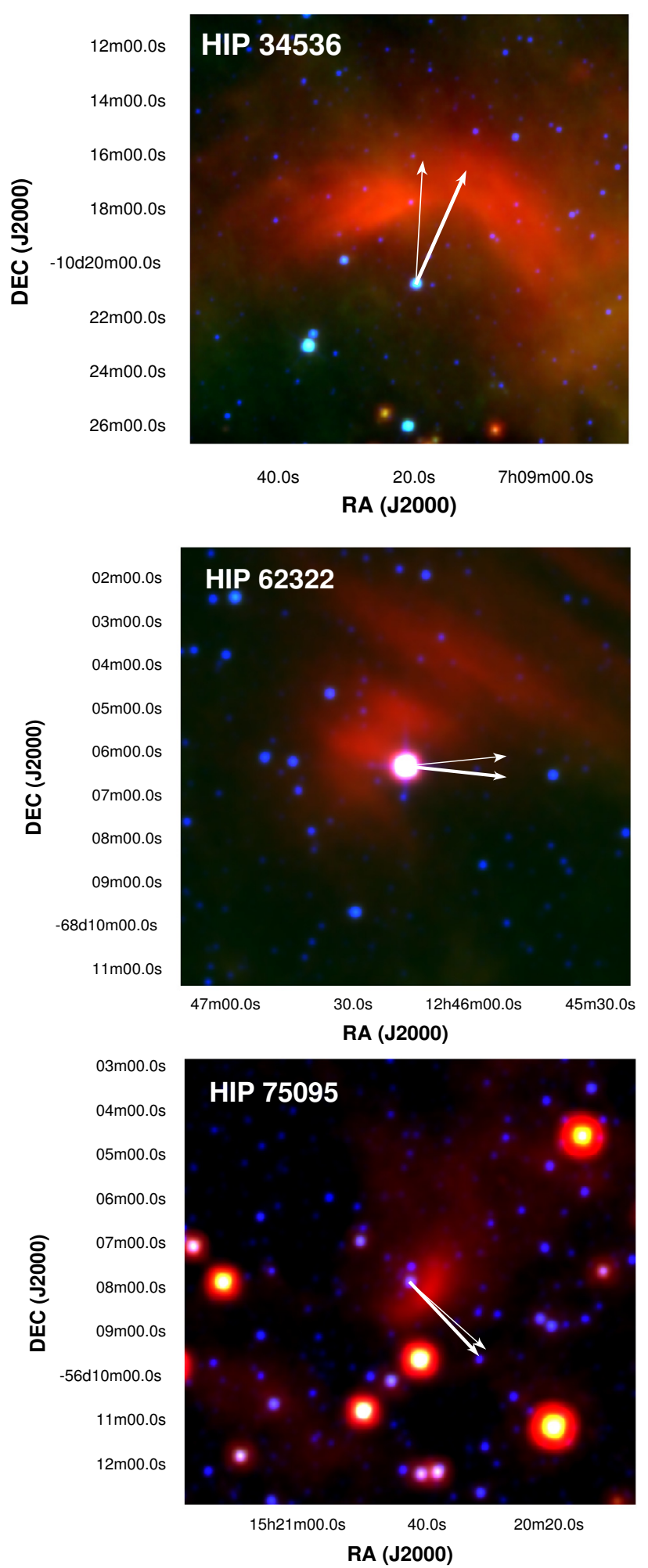

\section{Results}

\subsection{Group 1}

We searched for WISE and MSX data toward the 56 group- 1 targets, at all available wavelengths, with a field size of $1 \mathrm{sq}$ degree. About $55 \%$ of the targets have MSX data, and $70 \%$ have WISE data. Table 1 gives the Hipparcos number, alternative names, and the spectral type of the stars as in NC97, or 

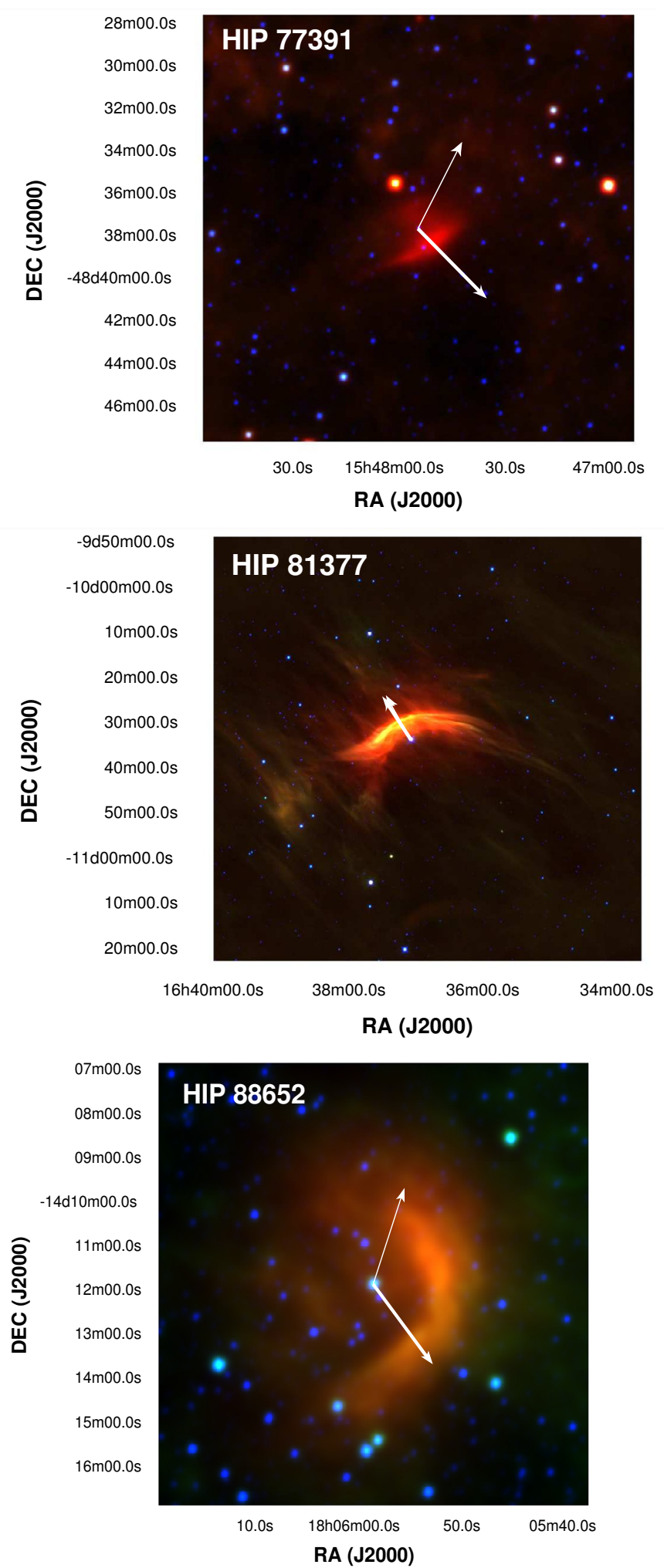

Fig. 4. Same as in Figs. 1-3, for another six OB stars.

updated from GOSC (Maíz Apellániz et al. 2004) whenever possible. Columns 4 and 5 list the results obtained with MSX and WISE. We identified several types of structures around the stars that are represented with different symbols in Table 1. The categories are: a point source at the position of the star, diffuse emission in the vicinity of the star, no emission, a bow-shaped emission feature, a bubble candidate, and extended emission in the field. A "?" symbol implies doubtful feature. The last column
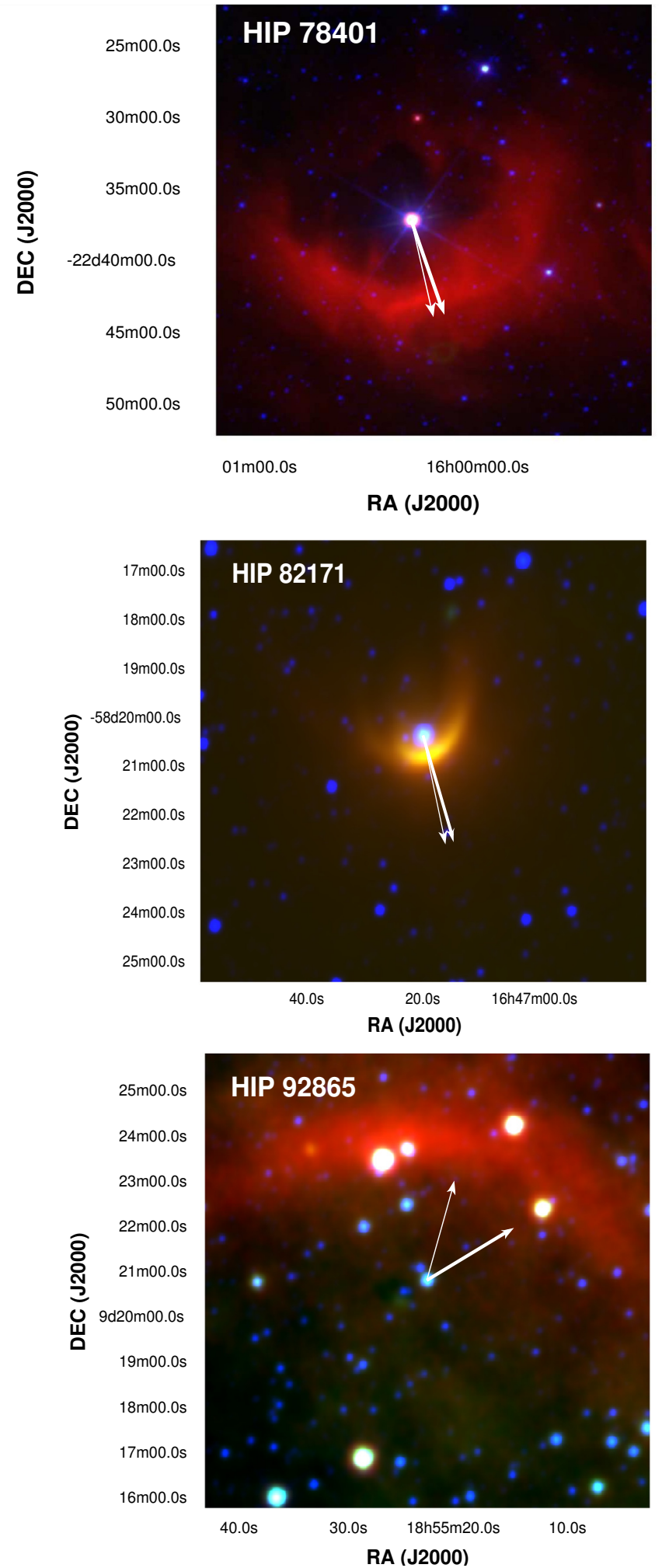

reproduces the results of NC97. Two special cases are noted in the table. Cappa et al. (2008) studied the environs of HD 92206, and identified an extended HII region. The star HD 36862 is very close to HD 36861 (O8III); they both belong to the rich cluster $\lambda$ Ori (Bouy et al. 2009), are not single isolated objects, and have a large spatial velocity. In both cases, a bow shock might be hidden and we discarded these two objects from our sample. 

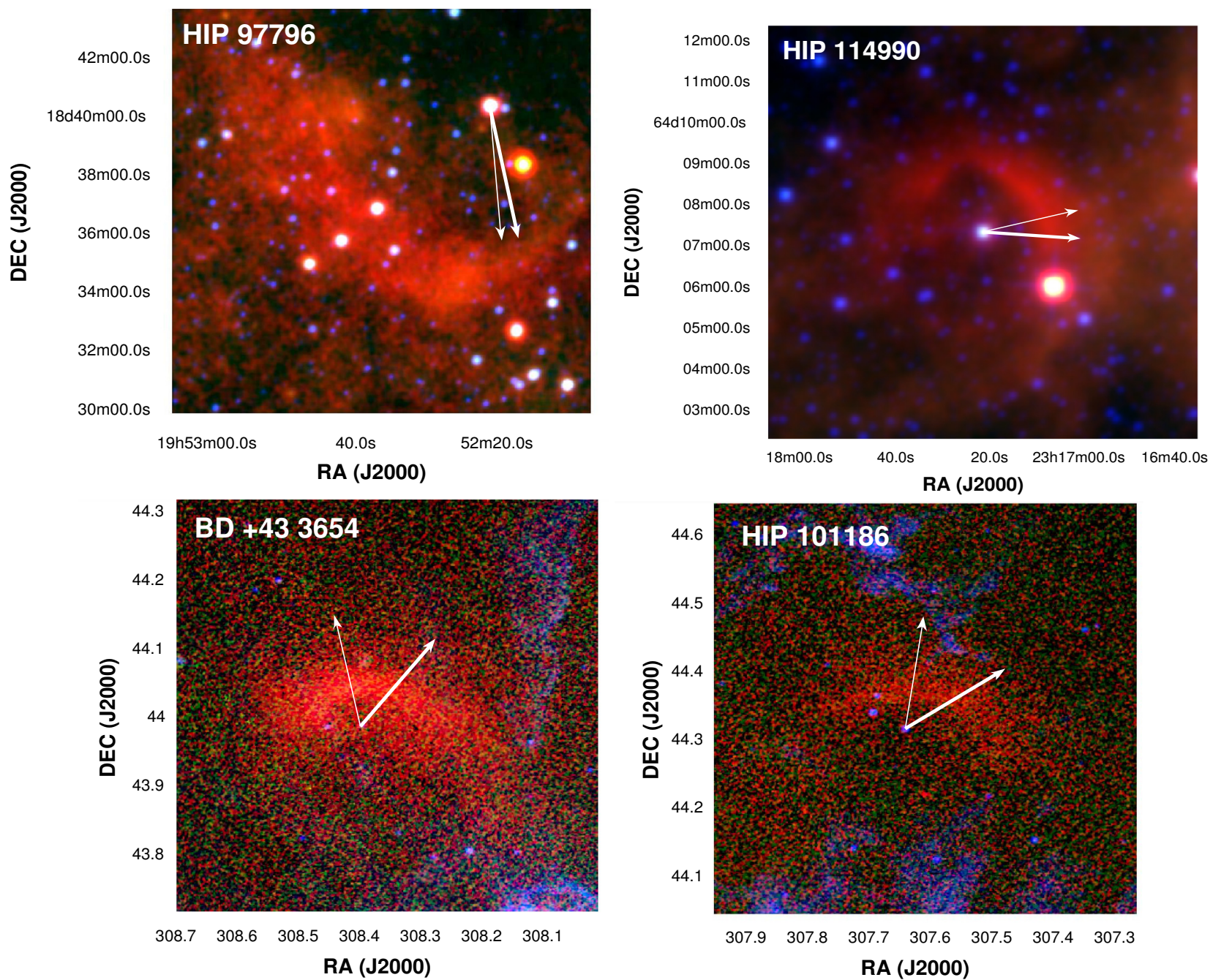

Fig. 5. WISE images of bow shock candidates as in Figs. 1-4, for HIP 97796 and 114990. MSX images for BD +43 3654 and HIP 101186 . Color mapping for MSX: blue $=8.3$ microns; green $=12.1$ microns; red $=21.3$ microns.

We found 18 bow-shock candidates (BS-C) out of the 56 stars of group 1 (Table 1). Of the $18 \mathrm{BS}-\mathrm{C}, 3$ of them were detected with MSX (one is the case of $\mathrm{BD}+43^{\circ} 3654$ ) and the other 15 with WISE. Out of a total of 18 BS-C, 14 were also classified as BS-C by NC97 (see Table 1). A total of 4 new BS-C were found here, related to HIP 24575, HIP 38430, HIP 77391, and HIP 114990. We believe that the different results between group 1 stars and NC97 are due to the high resolution and sensitivity of the more recent data.

\subsection{Group 2}

We list the 244 O-B2 runaway candidates extracted from Tetzlaff et al. (2010) in Table 2. We searched the WISE data to identify BS-C. The table is divided into two parts: the upper part contains those stars for which WISE data were released, and the lower portion where they were not.

We found a total of $17 \mathrm{BS}-\mathrm{C}$, marked with bold font in the top part of Table 2; seven of them had been identified in group 1 .

\subsection{The E-BOSS sample}

The 28 objects of the E-BOSS sample are shown in Figs. 1 to 5. In Table 3, the star name is given in the first column, the group membership in Col. 2, and Galactic coordinates in Cols. 3 and
4. Spectral types are in Col. 5. The distances, in Col. 6, were taken from several sources, namely Megier et al. (2009), Mason et al. (1998), Schilbach \& Röser (2008), Hanson (2003), and Thorburn et al. (2003), or derived from Hipparcos parallaxes (van Leeuwen 2007). The wind terminal velocities $v_{\infty}$ are either from Howarth et al. (1997) or derived using Table 3 of Prinja et al. (1990). To compute the stellar mass-loss rates $\dot{M}$, we used the routine described by Vink et al. (2001), and stellar parameters derived from standard models (e.g. Martins et al. 2005). The stellar tangential velocities $v_{\text {tg }}$ were taken from Tetzlaff et al. (2010), or derived from proper motions (van Leeuwen 2007, see last two columns of Table 3). Radial velocities $v_{\mathrm{r}}$ are from the Second Catalogue of Radial Velocities with Astrometric Data (Kharchenko et al. 2007).

In Figs. 1 to 5, we show the WISE and MSX images of the 28 BS-C. Superimposed on the images, we have plotted with arrows two directions, representing the proper motion of the star derived by van Leeuwen (2007), and that of the corrected proper motion after taking into account the Galactic rotation of the ISM at the location of the star (as done for example in Comerón \& Pasquali 2007; Moffat et al. 1998, 1999).

For each BS-C, we measured geometrical parameters, such as the spatial extent $l$, the width $w$, and the distance from the star to the midpoint of the bow shock structure $R$ (Table 4 , Cols. 2 to 7). 
Table 2. Star members of group 2.

\begin{tabular}{|c|c|c|c|c|c|c|c|c|c|c|c|}
\hline HIP & Sp.t. & HIP & Sp.t. & HIP & Sp.t. & HIP & Sp.t. & $\overline{\mathrm{HIP}}$ & Sp.t. & HIP & Sp.t. \\
\hline 278 & B2IV & 505 & O6pe & 1805 & BOIV & 2036 & B1V & 2599 & B1Ia & 4532 & B1II \\
\hline 4983 & B2IV-V & 5391 & B1V & 6027 & B2III & 8725 & O8V & 9538 & B1V & 10463 & B2IV-V \\
\hline 10527 & B0.5III & 10641 & B2Ib & 10849 & $\mathrm{~B} 2 \mathrm{~V}$ & 10974 & B2 & 11099 & $08.5 \mathrm{~V}$ & 11279 & B2Ia \\
\hline 11347 & B1Ib & 11394 & O6 & 11396 & B2 & 11473 & $09.5 \mathrm{~V}$ & 11792 & $\mathrm{O} 9 \mathrm{~V}$ & 11891 & $\mathrm{O} 5$ \\
\hline 12009 & B1Iab & 12293 & B2 & 13736 & B0II-III & 13924 & $\mathrm{O} 7 \mathrm{~V}$ & 14514 & B $1.5 \mathrm{~V}$ & 14626 & B1V \\
\hline 14777 & B2 & 14969 & B2IV & 15270 & B2.5IV-V & 16518 & B1V & 16566 & $\mathrm{O}$ & 17387 & B2V \\
\hline 18151 & B1III & 18350 & O9.5 & 18614 & O7.5Iab & 19218 & O8 & 21626 & B $2.5 \mathrm{~V}$ & 22061 & B $2.5 \mathrm{~V}$ \\
\hline 22461 & B1II-III & 23060 & B2V & 24072 & B2III & 24238 & B2V & 24575 & $09.5 \mathrm{~V}$ & 25923 & B0V \\
\hline 26064 & B2IV-V & 26397 & B $0.5 \mathrm{~V}$ & 26889 & BOII & 27204 & B1IV-V & 27850 & B1V & 27941 & O6 \\
\hline 28756 & B2V & 29201 & B0V & 29276 & B0.5IV & 29317 & B1V & 29321 & B2V & 29563 & B2V \\
\hline 29678 & B1V & 30961 & B2.5IV-V & 31766 & O9.5II & 31787 & B0IV & 32067 & O6 & 32300 & B0.5IV \\
\hline 32602 & O6 & 32947 & B2V & 33300 & B2V & 33754 & B1Ib & 34536 & O6 & 34924 & B2III \\
\hline 34986 & B0.5III & 35149 & B1.5III & 35951 & $\mathrm{~B} 2 \mathrm{~V}$ & 36369 & O6 & 36778 & $\mathrm{~B} 2 \mathrm{~V}$ & 37169 & O9.5Iab \\
\hline 38855 & B2V & 39172 & B $2.5 \mathrm{~V}$ & 40047 & O5p & 44685 & B2IV & 45880 & B2 & 46760 & B2V \\
\hline 48715 & B1Ib & 52670 & B $2.5 \mathrm{~V}$ & 54572 & B2V & 58748 & B1II & 61431 & B1Ib & 62322 & B2V \\
\hline 62829 & B0.5III & 63049 & B0IV & 63117 & O9Ib & 63170 & B0.5Ia & 63256 & B2V & 64272 & B1Ib \\
\hline 67663 & B2V & 68002 & B2.5IV-V & 68817 & B $0.5 \mathrm{~V}$ & 69892 & O8.5 & 69996 & B2.5IV & 70574 & B2IV \\
\hline 70877 & B2III & 71264 & B2V & 72438 & B $2.5 \mathrm{~V}$ & 72510 & O7.5 & 72710 & B2 & 74778 & $08.5 \mathrm{~V}$ \\
\hline 75095 & B2Ib & 75141 & B1.5IV & 75711 & B2II/III & 76013 & B1 & 76642 & B2III & 78145 & B0.5Ia \\
\hline 78582 & B2V & 79466 & B2III & 80782 & B1.5Iap & 80945 & B1Ia & 81100 & O6e & 81122 & B0Ia \\
\hline 81305 & O9Ia & 81377 & $09.5 \mathrm{~V}$ & 81696 & O7V & 82171 & B0Iab & 82378 & O9.5IV & 82691 & O7e \\
\hline 82775 & O8Iab... & 82783 & O9Ia & 83003 & O... & 83574 & B2Iab & 83635 & B1V & 84226 & B1Ib \\
\hline 84338 & B2III & 84401 & O9 & 84687 & B0V & 84745 & B2V & 85331 & O6.5III & 85530 & B2V \\
\hline 85885 & B2II & 87397 & B2III & 88004 & B1Iab & 88496 & B2V & 88584 & O6 & 88652 & O9.5Iab \\
\hline 88714 & B2Ib & 89743 & $09.5 \mathrm{~V}$ & 90610 & B2V & 90804 & B2V & 90950 & B0Ia/Iab & 91003 & $\mathrm{O} 7$ \\
\hline 91049 & B2II & 91599 & B $0.5 \mathrm{~V}$ & 92133 & B $2.4 \mathrm{~V}$ & 93118 & O7.5 & 93796 & B1Ib & 93934 & B2II \\
\hline 94934 & B2IV & 95408 & B2V & 96130 & B1.5III & 96362 & B2V & 97246 & B1Ia & 97545 & B1V \\
\hline 97679 & $\mathrm{~B} 2.5 \mathrm{~V}$ & 117514 & $\mathrm{~B} 2 \mathrm{~V}$ & & & & & & & & \\
\hline 3013 & B2 & 38518 & B0.5Ib & 39429 & O8Iaf & 39776 & B2.5III & 40341 & $\mathrm{~B} 2 \mathrm{~V}$ & 41168 & B2IV \\
\hline 41463 & B2V & 41878 & B $1.5 \mathrm{Ib}$ & 42316 & B1Ib & 42354 & B2III & 43158 & B0II/III & 43868 & B1Ib \\
\hline 44251 & B2.5V & 44368 & B0.5Ib & 46950 & B1.5IV & 47868 & B0IV & 48469 & B1V & 48527 & B2V \\
\hline 48730 & B2IV-V & 48745 & B2III & 49608 & B1III & 49934 & B2IV & 50899 & B0Iab/Ib & 51624 & B1Ib \\
\hline 52526 & B0Ib & 52849 & $\mathrm{O} 9 \mathrm{~V}$ & 52898 & B2III & 54179 & B1Iab & 54475 & O9II & 58587 & B2IV \\
\hline 61958 & Op & 65388 & B2 & 74368 & B0 & 89902 & B2V & 94716 & B1III-III & 97045 & B0V \\
\hline 97845 & B0.5III & 98418 & $\mathrm{O} 7$ & 98661 & B1Iab & 99283 & B0.5IV & 99303 & B $2.5 \mathrm{~V}$ & 99435 & B $0.5 \mathrm{~V}$ \\
\hline 99580 & O5e & 99953 & B1V & 100088 & B $1.5 \mathrm{~V}$ & 100142 & B2V & 100314 & B1.5Ia & 100409 & B1Ib \\
\hline 101186 & O9.5Ia & 101350 & B0V & 102999 & BOIV & 103763 & B2V & 104316 & O9 & 104548 & B1V \\
\hline 104579 & B1V & 104814 & B $0.5 \mathrm{~V}$ & 105186 & $\mathrm{O} 8$ & 105912 & B2II & 106620 & B2V & 106716 & B2V \\
\hline 107864 & Op & 108911 & B2Iab & 109051 & B2.5III & 109082 & B2V & 109311 & B1V & 109332 & B2III \\
\hline 109556 & B1II & 109562 & O9Ib & 109996 & B1II & 110025 & B2III & 110287 & B1V & 110362 & B0.5IV \\
\hline 110386 & B2IV-V & 110662 & B1.5IV-V & 110817 & B0.5Ib & 111071 & B0IV & 112482 & B1II & 112698 & B1V \\
\hline 114482 & O9.5Iab & 114685 & $\mathrm{O} 7$ & & & & & & & & \\
\hline
\end{tabular}

Notes. Top: stars with WISE observations (164 stars). Bottom: stars without WISE observations (80 stars). The spectral types are from Tetzlaff et al. (2010). In bold font: the 17 bow shock candidates detected, see text.

The ISM ambient density in the vicinity of the star $n_{\text {ISM }}$ can be estimated using the expression that gives the so-called stagnation radius $R_{0}$ (see Wilkin 1996, for definition and details)

$R_{0}=\sqrt{\frac{\dot{M} v_{\infty}}{4 \pi \rho_{\mathrm{a}} v_{*}^{2}}}$,

where the ambient medium density is $\rho_{\mathrm{a}}=\mu n_{\mathrm{ISM}}$ and $v_{*}$ is the spatial stellar velocity. We estimated the volume density of the ISM in $\mathrm{H}$ atoms at the bow shock position assuming $R_{0} \sim R$, a mass per $\mathrm{H}$ atom $\mu=2.3 \times 10^{-24} \mathrm{~g}$, and the helium fractional abundance $Y=0.1$. The values obtained for $n_{\text {ISM }}$ are given in column 8 of Table 4 , and should be interpreted with caution. In many cases, the width of the bow shock is substantial compared to $R$, which adds an uncertainty to the $R_{0}$ values used in the last equation.

There are additional factors that might affect the values of $R_{0}$ and $n_{\mathrm{ISM}}$, such as errors in the mass-loss rate due to clumping, in addition to the potential source of errors in the parameters used, as described above.

\section{Statistics}

In Figs. 6 and 7, we present the $(l, b)$ distribution of group 1 and group 2 stars, showing those with and without bow shocks. There does not seem to be any preferential location for stars with bow shocks. We note that there are no bow shocks at high latitudes, but that the small number of stars there means we cannot say anything conclusive.

Figures 8 and 9 show the occurrence of bow shocks as a function of spectral type for each group. We might have expected more detections of bow shocks from the more massive, earliertype, or fastest stars, which is not seen. The number of stars in each subtype grows with later spectral types, probably reflecting that there is no strong bias in the Group 2 stellar sample.

Figures 10 and 11 show that bow shocks were detected around both the lower mass stars and the youngest stars, but the 
A\&A 538, A108 (2012)

Table 3. List of the E-BOSS bow shock candidates and corresponding stellar parameters.

\begin{tabular}{|c|c|c|c|c|c|c|c|c|c|c|c|}
\hline Star & Group & $\begin{array}{c}l \\
{\left[{ }^{\circ}\right]}\end{array}$ & $\begin{array}{c}b \\
{\left[{ }^{\circ}\right]}\end{array}$ & Spectral type & $\begin{array}{c}d \\
{[\mathrm{pc}]}\end{array}$ & $\begin{array}{c}v_{\infty} \\
{\left[\mathrm{km} \mathrm{s}^{-1}\right]}\end{array}$ & $\begin{array}{c}\dot{M} \times 10^{6} \\
{\left[M_{\odot} \mathrm{yr}^{-1}\right]}\end{array}$ & $\begin{array}{c}v_{\mathrm{tg}} \\
{\left[\mathrm{km} \mathrm{s}^{-1}\right]}\end{array}$ & $\begin{array}{c}v_{\mathrm{r}} \\
{\left[\mathrm{km} \mathrm{s}^{-1}\right]}\end{array}$ & $\begin{array}{c}\mu_{\alpha} \cos \delta \\
{\left[\mathrm{mas} \mathrm{yr}^{-1}\right]}\end{array}$ & $\begin{array}{c}\mu_{\delta} \\
{\left[\mathrm{mas} \mathrm{yr}^{-1}\right]}\end{array}$ \\
\hline HIP 2036 & 2 & 120.9137 & +09.0357 & O9.5III+B1V & $757 \pm 161^{a}$ & [1200] & 0.48 & 15.2 & -5 & -1.66 & 1.90 \\
\hline HIP 2599 & 1,2 & 120.8361 & +00.1351 & B1 Iae & $1457 \pm 300^{\mathrm{a}}$ & 1105 & 0.12 & 26.2 & -2.3 & 3.65 & -2.07 \\
\hline HIP 11891 & 2 & 134.7692 & +01.0144 & O5 V((f)) & ( 900 ) & 2810 & 1.10 & 11.9 & -48 & 0.03 & -2.16 \\
\hline HIP 16518 & 2 & 156.3159 & -16.7535 & B1 V & $(650)$ & [500] & 0.006 & 47.3 & 25 & -8.28 & 3.44 \\
\hline HIP 17358 & 1 & 150.2834 & -05.7684 & B5 III & ( 150$)$ & [500] & $<0.001$ & [35] & 4 & 25.58 & -43.06 \\
\hline HIP 22783 & 1 & 144.0656 & +14.0424 & O9.5 Ia & $1607 \pm 275^{\mathrm{a}}$ & 1590 & 0.25 & [52] & 6.1 & -0.13 & 6.89 \\
\hline HIP 24575 & 2 & 172.0813 & -02.2592 & $09.5 \mathrm{~V}$ & $548 \pm 68^{a}$ & [1200] & 0.1 & 140.0 & 59.1 & -3.58 & 43.73 \\
\hline HIP 25923 & 2 & 210.4356 & -20.9830 & $\mathrm{~B} 0 \mathrm{~V}$ & ( 900 ) & [1000] & 0.06 & 16.8 & 17.4 & -0.10 & -4.87 \\
\hline HIP 26397 & 2 & 174.0618 & +01.5808 & B0.5 V & ( 350$)$ & [750] & 0.014 & 11.9 & -19 & 0.88 & -3.61 \\
\hline HIP 28881 & 1 & 164.9727 & +12.8935 & $\mathrm{O} 8 \mathrm{Vn}$ & $1500^{b}$ & 2070 & 0.03 & [17] & 5 & -0.82 & -1.49 \\
\hline HIP 29276 & 1,2 & 263.3029 & -27.6837 & B1/2 III & ( 400 ) & [600] & $<0.001$ & 9.2 & 30.6 & -4.90 & 7.41 \\
\hline HIP 31766 & 2 & 210.0349 & -02.1105 & O9.7 Ib & $1414 \pm 28^{a}$ & 1590 & 1.07 & 6.7 & 58.4 & -0.34 & -0.83 \\
\hline HIP 32067 & 1,2 & 206.2096 & +00.7982 & $05.5 \mathrm{~V}((\mathrm{f}))+\ldots$ & $2117 \pm 367^{\mathrm{a}}$ & 2960 & 0.13 & 23.4 & 31 & 0.84 & 2.55 \\
\hline HIP 34536 & 1,2 & 224.1685 & -00.7784 & $\mathrm{O} 6.5 \mathrm{~V}(\mathrm{f}))+\ldots$ & $1293 \pm 206^{\mathrm{a}}$ & 2456 & 0.19 & 14.3 & 58 & -1.96 & 4.40 \\
\hline HIP 38430 & 1 & 243.1553 & +00.3630 & $\mathrm{O} 6 \mathrm{Vn}+\ldots$ & ( & [2570] & 0.7 & [13] & 28 & -3.04 & -0.38 \\
\hline HIP 62322 & 2 & 302.4492 & -05.2412 & $\mathrm{~B} 2.5 \mathrm{~V}$ & ( 150$)$ & {$[300]$} & 0.006 & 4.5 & 42 & -41.97 & -8.89 \\
\hline HIP 72510 & 1,2 & 318.7681 & +02.7685 & O6.5III(n)(f) & $(350)$ & [2545] & 0.27 & 7.4 & -74 & -7.49 & -5.15 \\
\hline HIP 75095 & 2 & 322.6802 & +00.9060 & B1Iab/Ib & ( 800$)$ & [1065] & 0.14 & 28.6 & 4 & -8.42 & -9.18 \\
\hline HIP 77391 & 1 & 330.4212 & +04.5928 & O9 I & $(800)$ & [1990] & 0.25 & [19] & 15 & -4.63 & -1.84 \\
\hline HIP 78401 & 1 & 350.0969 & +22.4904 & B0.2 IVe & $224 \pm 24^{\mathrm{a}}$ & [1100] & 0.14 & [38] & -7 & -10.21 & -35.41 \\
\hline HIP 81377 & 1,2 & 006.2812 & +23.5877 & O9.5 Vnn & $222 \pm 22^{\mathrm{a}}$ & [1500] & 0.02 & 24.4 & -15 & 15.26 & 24.79 \\
\hline HIP 82171 & 2 & 329.9790 & -08.4736 & B0.5 Ia & $845 \pm 120^{\mathrm{a}}$ & 1345 & 0.09 & 65.7 & -53.3 & -4.64 & -20.28 \\
\hline HIP 88652 & 2 & 015.1187 & +03.3349 & B0 Ia & ( 650 ) & [1535] & 0.5 & 8.2 & 30 & -1.05 & -1.38 \\
\hline HIP 92865 & 1 & 041.7070 & +03.3784 & O8 Vnn & $(350)$ & [1755] & 0.04 & [2] & -41 & -0.78 & 0.46 \\
\hline HIP 97796 & 1 & 056.4824 & -04.3314 & O7.5 Iabf & $2200^{c}$ & [1980] & 0.50 & [110] & 9 & -2.03 & -10.30 \\
\hline HIP 101186 & 1 & 082.3557 & +02.9571 & O9.7 Ia & $1486 \pm 402^{\mathrm{a}}$ & [1735] & 0.23 & 22.3 & -28 & -2.37 & 1.37 \\
\hline $\mathrm{BD}+433654$ & 1 & 082.4100 & +02.3254 & O4 If & $1450^{\mathrm{d}}$ & [2325] & 6.5 & {$[14]$} & -66.2 & -0.44 & 1.3 \\
\hline HIP 114990 & 1 & 112.8862 & +03.0998 & B0 II & $1400^{\mathrm{e}}$ & [1400] & 0.6 & [52] & -125.3 & -7.86 & -0.71 \\
\hline
\end{tabular}

Notes. Galactic coordinates: taken from Simbad. Spectral types: for B-type stars from the Simbad database, for O-type stars GOS Catalog.

References. References for the distance values: (a) Megier et al. (2009), (b) Mason et al. (1998), (c) Schilbach \& Roeser (2008), (d) Hanson (2003), (e) Thorburn et al. (2003); distances in brackets: derived from Hipparcos (van Leeuwen 2007) parallaxes. Terminal velocities in square brackets: from Howarth et al. (1997), otherwise inter- or extrapolated from Prinja et al. (1990). Mass-loss rates: derived from Vink et al. (2001). Tangential velocities in brackets derived from proper motions (van Leeuwen 2007), otherwise from Tetzlaff et al. (2010). Radial velocities are from the Second Catalog of Radial Velocities with Astrometric Data (Kharchenko et al. 2007).

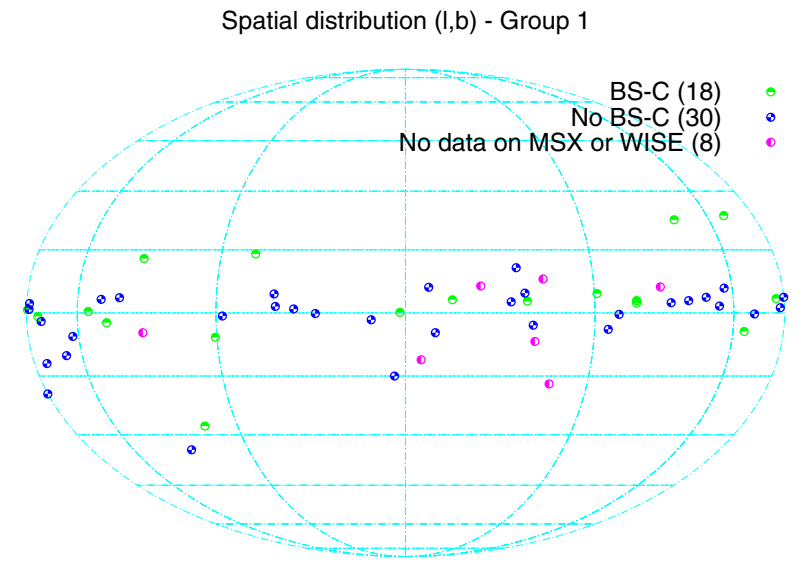

Fig. 6. Distribution on the $(l, b)$ plane of group-1 stars.

very small numbers in many of the bins prevents us to draw any clear conclusion.

\section{Discussion}

The E-BOSS sample introduced here constitutes the most substantial sample of stellar bow shocks and should provide a

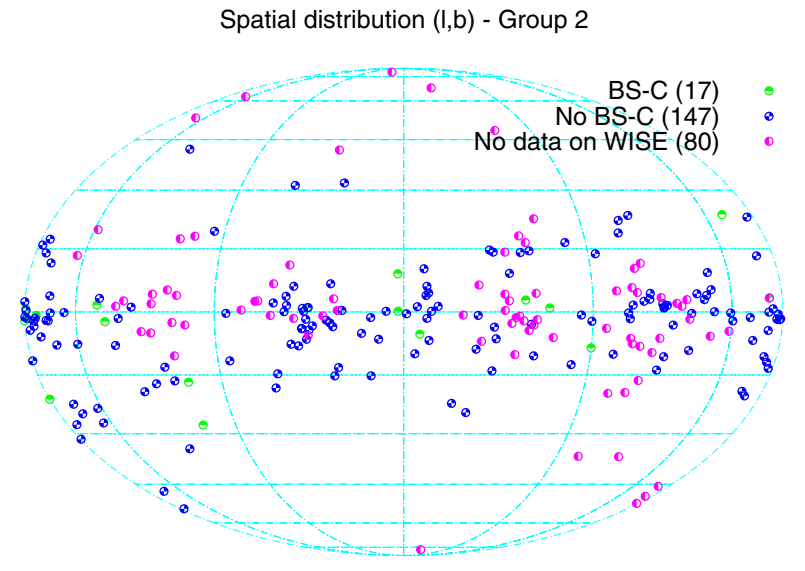

Fig. 7. Distribution on the $(l, b)$ plane of group-2 stars.

reliable basis to more detailed studies of the structure and formation of bow shocks.

For group 1, the availability of MSX and WISE images has enabled us to improve previous results, which relied on the IRAS data. Some structures were identified as bow shocks, others rejected, and in some cases new bow shocks were revealed. One interesting example is the feature around HD 34078 
Table 4. Observational parameters of the bow shock candidates obtained from the images of MSX and WISE.

\begin{tabular}{|c|c|c|c|c|c|c|c|c|}
\hline Star & $\overline{l l}$ & $\begin{array}{c}w \\
{[\operatorname{arcmin}]}\end{array}$ & $\bar{R}$ & $\bar{l}$ & $\begin{array}{c}w \\
{[\mathrm{pc}]}\end{array}$ & $R$ & $\begin{array}{c}n_{\mathrm{ISM}} \\
{\left[\mathrm{cm}^{-3}\right]}\end{array}$ & Comments \\
\hline HIP 2036 & 4.5 & 1.3 & 1 & 0.99 & 0.29 & 0.22 & 130 & Emission-line star \\
\hline HIP 2599 & 9 & 1.3 & 3 & 3.81 & 0.55 & 1.27 & 0.4 & Emission-line star \\
\hline HIP 11891 & 4 & 1 & 1 & 1.05 & 0.26 & 0.26 & 3 & Star in cluster \\
\hline HIP 16518 & 4 & 1 & 0.7 & 0.76 & 0.19 & 0.13 & 0.2 & Variable star \\
\hline HIP 17358 & 3 & 1 & 1 & 0.13 & 0.04 & 0.04 & 600 & Variable star \\
\hline HIP 22783 & 33 & 10 & 10 & 15.43 & 4.67 & 4.67 & 0.02 & Emission-line star \\
\hline HIP 24575 & 2 & 0.5 & 0.4 & 0.32 & 0.08 & 0.06 & 3 & Double or multiple star \\
\hline HIP 25923 & 4 & 1 & 1.5 & 1.05 & 0.26 & 0.39 & 1 & Variable star \\
\hline HIP 26397 & 3 & 1 & 1 & 0.31 & 0.10 & 0.10 & 2 & Star \\
\hline HIP 28881 & 9 & 1.5 & 3 & 5.54 & 0.92 & 1.85 & 0.3 & Double or multiple star \\
\hline HIP 29276 & 5 & 2 & 2 & 0.58 & 0.23 & 0.23 & 0.003 & Eclipsing binary of beta Lyr type \\
\hline HIP 31766 & 5 & 2 & 2 & 2.06 & 0.82 & 0.82 & 0.03 & Double or multiple star \\
\hline HIP 32067 & 13 & 2.5 & 3 & 8.01 & 1.54 & 1.85 & 0.1 & Emission-line star \\
\hline HIP 34536 & 12 & 3 & 4 & 4.51 & 1.13 & 1.50 & 0.01 & HII (ionized) region \\
\hline HIP 38430 & 2 & 0.5 & 0.5 & 0.52 & 0.13 & 0.13 & 60 & Emission-line star \\
\hline HIP 62322 & 4 & 1.2 & 1 & 0.17 & 0.05 & 0.04 & 0.02 & Double or multiple star \\
\hline HIP 72510 & 4.5 & 0.8 & 1.5 & 0.46 & 0.08 & 0.15 & 0.2 & Emission-line star \\
\hline HIP 75095 & 1.5 & 0.5 & 0.5 & 0.35 & 0.12 & 0.12 & 40 & Star \\
\hline HIP 77391 & 4 & 1 & 1 & 0.93 & 0.23 & 0.23 & 30 & Star \\
\hline HIP 78401 & 25 & 2 & 6 & 1.63 & 0.13 & 0.39 & 2 & Double or multiple star \\
\hline HIP 81377 & 22 & 2 & 5 & 1.42 & 0.13 & 0.32 & 1 & Be star \\
\hline HIP 82171 & 2 & 0.5 & 0.7 & 0.49 & 0.12 & 0.17 & 1 & Star \\
\hline HIP 88652 & 6 & 1 & 1.5 & 1.13 & 0.19 & 0.28 & 2 & Star \\
\hline HIP 92865 & 11 & 1 & 3 & 1.12 & 0.10 & 0.31 & 0.003 & Eclipsing binary of beta Lyr type \\
\hline HIP 97796 & 13 & 2.5 & 6 & 8.32 & 1.60 & 3.84 & 0.02 & Spectroscopic binary \\
\hline HIP 101186 & 19 & 2.5 & 4 & 8.21 & 1.08 & 1.73 & 0.1 & Emission-line star \\
\hline $\mathrm{BD}+433654$ & 12 & 3 & 3.5 & 5.06 & 1.27 & 1.48 & 0.2 & Star \\
\hline HIP 114990 & 3.5 & 0.75 & 1.5 & 1.43 & 0.31 & 0.61 & 0.05 & Star \\
\hline
\end{tabular}

Notes. Columns (2) to (7): length $(l)$ and width $(w)$ of the bow shock structure, and distance $(R)$ from the star to the midpoint of the bow shock, in angular and linear units. Column (8): the ambient density $n_{\text {ISM }}$ (see text). The descriptions in the last column are taken from the Simbad database.

(HIP 24575). The IRAS data, discussed in NC97, revealed an excess of emission at $60 \mu \mathrm{m}$, but no discernible bow shock. With the WISE bands, two structures can be clearly seen (Fig. 2). There are filaments around the star mainly at longer wavelengths (red = 22.2 microns), and a typical bow-shaped feature becomes visible in the longer bands (red $=22.2$ microns and green $=$ 12.1 microns) in the direction of the stellar motion. Another example from group 1 is the object related to the B5 III star HD 22928 (HIP 17358). The combination of low mass-loss rate and wind terminal velocity, with a high stellar velocity leads to the formation of a bow shock very close to the star. This situation results in a very large derived value of the ISM density (Table 4). The stellar motion is dominated by the tangential velocity, and is a nearby star. These help us to more clearly resolve the bow shock.

The strategy we adopted here for group 2, beginning with the early-type runaways, which are most likely to form bow shocks, has been successful in identifying a significant number of new bow shocks. These results support the hypothesis that some runaway stars (around 10\%) produce detectable bow shocks. There are many possible reasons why a detectable bow shock is not formed, such as a low-density ISM, an extremely high stellar velocity, or a low mass-loss rate, the inclination of the stellar velocity vector with respect to the plane of the sky, and confusion with strong field sources.

The current release of WISE data covers around 57 per cent of the sky, but covers a slightly larger fraction of the Galactic plane. For our group 2 sample (244 stars), around 67 per cent of the stars were covered in the first WISE data release (164 stars). A total of 17 of these were found to have bow shocks (a detection

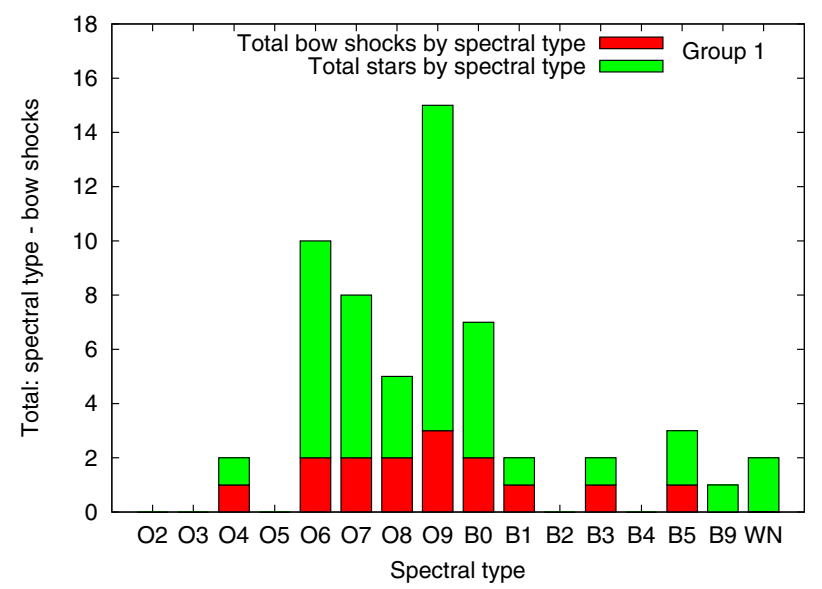

Fig. 8. Distribution of group-1 stars by spectral types.

rate of $\sim 10$ per cent). On the basis of these statistics, we would expect to find around 8 additional bow shock candidates in the remaining 80 group 2 sources.

Returning to the detected BS-C, several bow shocks in both group 1 and group 2 show a complex layered structure, such as HD 30614 (HIP 22783), HD 42933 (HIP 29276), and HD 15629 (HIP 11891). In many cases, but not all, the structures are aligned with the stellar velocity. An example of a misaligned bow shock candidate is HD 36512 (HIP 25923). We include this object in the sample because at this stage we lack any precise knowledge of the true stellar velocity. 


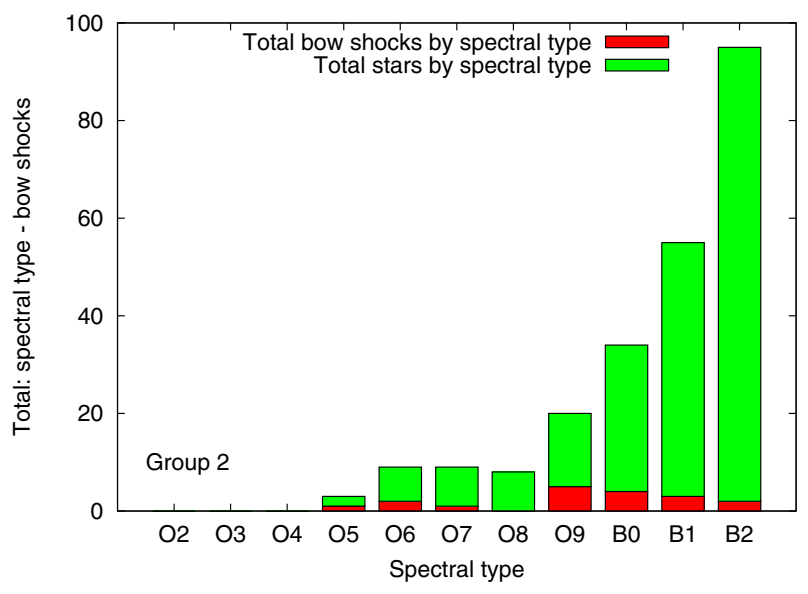

Fig. 9. Distribution of group-2 stars by spectral types.

HD 48099 (HIP 32076) is maybe the most classic example of a bow shock, and this object will be the subject of a future detailed study that will include modeling of the infrared emission in the formation of a bow shock (del Valle et al., in prep.).

With this sample, we will be able to investigate the IR luminosity and dust temperature and compare the scaling of these quantities with stellar parameters (van Buren \& McCray 1998), and also detectability (Stevens et al., in prep.).

The current sample is insufficiently large to distinguish properties according to stellar luminosity class, binarity status, or particular stellar classes, such as Of-type stars. However, when new data are released, the situation will improve.

\section{Summary and prospects}

We have discovered a significant number of bow shock candidates around early-type runaway stars providing a higher quality sample of bow shocks, the E-BOSS sample. For the set, we have determined a number of parameters of these objects. We have found no strong trends concerning the frequency of bow shocks with stellar mass, position, age, velocity, and spectral type.

In terms of future work, extending our study to later spectral types will allow us to systematically search for and investigate radiative bow shocks (see for example Gáspár et al. 2008). The extensive Tetzlaff et al. (2010) database will also serve as a starting point for that study. We will also incorporate in forthcoming E-BOSS versions other runaway databases together with individual objects from the literature.

Detailed studies of individual objects will help us to more clearly understand the stellar winds of the bow-shock producer stars, the medium in which they travel, and the stellar history, among other things. We plan to continue our studies of individual objects in the E-BOSS sample, as well as statistical studies of the global sample.

Acknowledgements. C. S. Peri and P.B. are supported by the ANPCyT PICT2007/00848. P.B. also acknowledges support from CONICET PIP 0078 and UNLP G093 projects, and thanks the School of Physics and Astronomy of the University of Birmingham for kind hospitality. This publication makes uses of the NASA/IPAC Infrared Science Archive, which is operated by the Jet Propulsion Laboratory, California Institute of Technology, under contract with the National Aeronautics and Space Administration, the SIMBAD database, operated at CDS, Strasbourg, France, and data products from the Widefield Infrared Survey Explorer, which is a joint project of the University of California, Los Angeles, and the Jet Propulsion Laboratory/California Institute of Technology, funded by the National Aeronautics and Space Administration. C. S. Peri is grateful to M. V. del Valle for a discussion on theoretical issues.

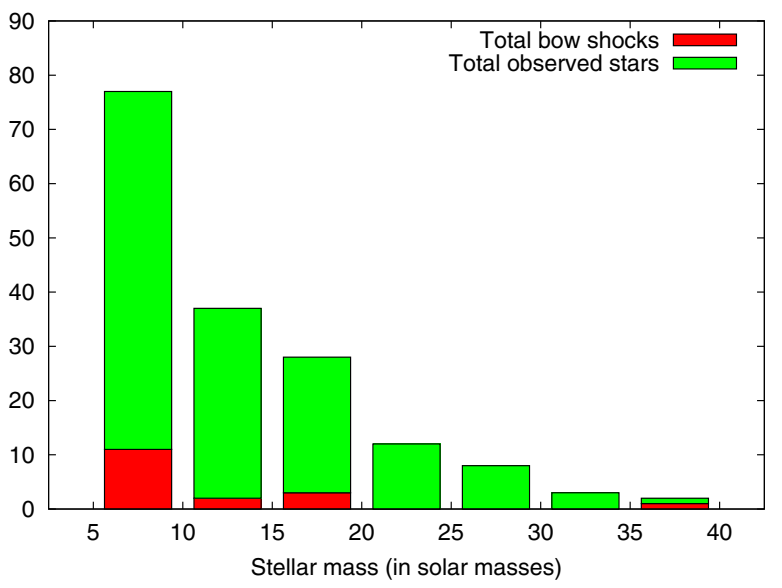

Fig. 10. Distribution of group- 2 stars by stellar mass $\left(5 M_{\odot}\right.$ binning).

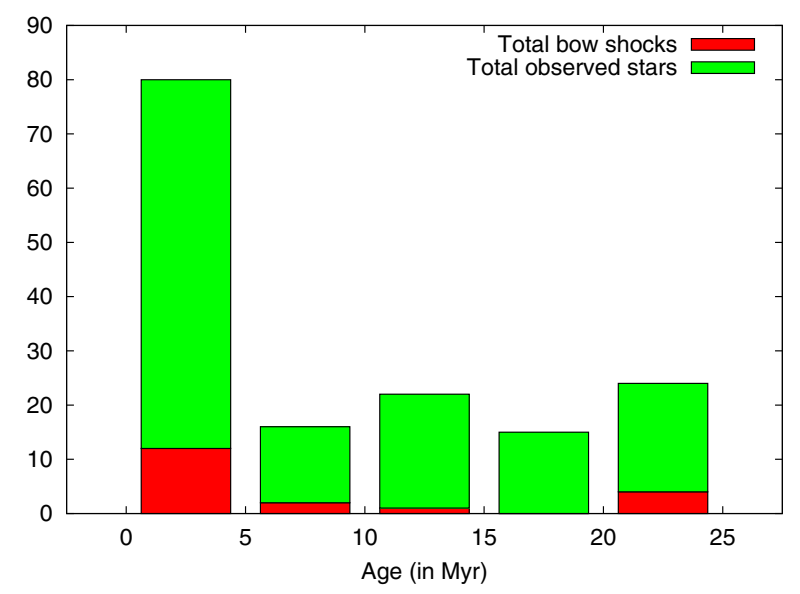

Fig. 11. Distribution of group-2 stars by age (5 Myr binning).

We also thank an unknown A\&A referee for the comments and suggestions that have improved the article.

\section{References}

Arnal, M. E., Cichowolski, S., Pineault, S., Testori, J. C., \& Cappa, C. 2011, A\&A, 532, A9

Benaglia, P., Romero, G. E., Martí, J., Peri, C. S., \& Araudo, A. T. 2010, A\&A, 517, L10

Blauuw, A. 1961, BAN, 15, 265

Bouy, H., Huélamo, N., Barrado y Navascués, D., et al. 2009, A\&A, 504, 199

Brown, D., \& Bomans, D. J. 2005, A\&A, 439, 183

Cappa, C., Niemela, V. S., Amorín, R., \& Vasquez, J. 2008, A\&A, 477, 173

Comerón, F., \& Kaper, L. 1998, A\&A, 338, 273

Comerón, F., \& Pasquali, A. 2007, A\&A, 467, L23

Condon, J. J., Cotton, W. D., Greisen, E. W., et al. 1998, AJ, 115, 1693

Dennison, B., Topasna, G., \& Simonetti, J. H. 1997, ApJ, 474, L31

Dgani, R., Van Buren, D., \& Noriega-Crespo, A. 1996a, ApJ, 461, 927

Dgani, R., Van Buren, D., \& Noriega-Crespo, A. 1996b, ApJ, 461, 372

Gáspár, A., Su, K. Y. L., Rieke, G. H., et al. 2008, ApJ, 672, 974

Gaustad, J. E., McCullough, P. R., Rosing, W., \& Van Buren, D. 2001, PASP, 113,1326

Gies, D. R., \& Bolton, C. T. 1986, ApJS, 61, 419

Gvaramadze, V. V., \& Bomans, D. J. 2008, A\&A, 485, L29

Gvaramadze, V. V., Röser, S., Scholz, R.-D., \& Schilbach, E. 2011a, A\&A, 529, A14

Gvaramadze, V. V., Kniazev, A. Y., Kroupa, P., \& Oh, S. 2011b, A\&A, 535, A29 Hanson, M. M. 2003, ApJ, 597, 957

Hoogerwerf, R., de Bruijne, J. H. J., \& de Zeeuw, P. T. 2000, ApJ, 544, L133 Howarth, I. D., Siebert, K. W., Hussain, G. A. J., \& Prinja, R. K. 1997, MNRAS 284, 265

Kaper, L., Van Loon, J. Th., Augusteijn, T., et al. 1997, ApJ, 479, L153 
C. S. Peri et al.: The extensive stellar BOw shock survey

Kharchenko, N. V., Scholz, R.-D., Piskunov, A. E., Röser S., \& Schilbach, E. 2007, AN, 328, 889

Kobulnicky, H. A., Gilbert, I. J., \& Kiminki, D. C. 2010, ApJ, 710, 549

Maíz-Apellániz, J., Walborn, N. R., Galue, H. A., \& Wei, L. H. 2004, ApJS, 151, 103

Martins, F., Schaerer, D., \& Hillier, D. J. 2005, A\&A, 436, 1049

Mason, B. D., Gies, D. R., Hartkopf, W. I., et al. 1998, AJ, 115, 821

Megier, A., Strobel, A., Galazutdinov, G. A., \& Krelowski, J. 2009, A\&A, 507, 833

Moffat, A. F. J., Marchenko, S. V., Seggewiss, W., et al. 1998, A\&A, 331, 949 Moffat, A. F. J., Marchenko, S. V., Seggewiss, W., et al. 1999, A\&A, 345, 321

Noriega-Crespo, A., Van Buren, D., \& Dgani, R. 1997, AJ, 113, 780

Perryman, M. A. C., Lindegren, L., Kovalevsky, J., et al. 1997, A\&A, 323, L49
Poveda, A., Ruiz, J., \& Allen, C. 1967, BOTyT, 4, 86

Povich, M. S., Benjamin, R. A., Whitney, B. A., et al. 2008, ApJ, 689, 242

Prinja, R. K., Barlow, M. J., \& Howarth, I. D. 1990, AJ, 361, 607

Schilbach, E., \& Röser, S. 2008, A\&A, 489, 105

Tetzlaff, N., Neuhäuser, R., \& Hohle, M. M. 2010, MNRAS, 410, 190

Thorburn, J. A., Hobbs, L. M., McCall, B. J., et al. 2003, ApJ, 584, 339

Van Buren, D., \& McCray, R. 1988, ApJ, 329, L93

Van Buren, D., Noriega-Crespo, A., \& Dgani, R. 1995, AJ, 110, 2914

Van Leeuwen, F. 2007, A\&A, 474, 653

Vink, J. S., de Koter, A., \& Lamers, H. J. G. L. M. 2001, A\&A, 369, 574

Wilkin, F. P. 1996, ApJ, 459, L31

Wilkin, F. P. 2000, ApJ, 532, 400

Zwicky, F. 1957, Morphological Astronomy (Berlin: SpringerVerlag) 